\title{
Cosmic String Interpretation of NANOGrav Pulsar Timing Data
}

\author{
John Ellis $\oplus^{1,2,3, *}$ and Marek Lewicki® ${ }^{1,4, \dagger}$ \\ ${ }^{1}$ Kings College London, Strand, London WC2R 2LS, United Kingdom \\ ${ }^{2}$ Theoretical Physics Department, CERN, 1211 Geneva 23, Switzerland \\ ${ }^{3}$ National Institute of Chemical Physics \& Biophysics, Rävala 10, 10143 Tallinn, Estonia \\ ${ }^{4}$ Faculty of Physics, University of Warsaw ul. Pasteura 5, 02-093 Warsaw, Poland
}

(Received 17 September 2020; accepted 15 December 2020; published 28 January 2021; corrected 15 April 2021)

Pulsar timing data used to provide upper limits on a possible stochastic gravitational wave background (SGWB). However, the NANOGrav Collaboration has recently reported strong evidence for a stochastic common-spectrum process, which we interpret as a SGWB in the framework of cosmic strings. The possible NANOGrav signal would correspond to a string tension $G \mu \in\left(4 \times 10^{-11}, 10^{-10}\right)$ at the $68 \%$ confidence level, with a different frequency dependence from supermassive black hole mergers. The SGWB produced by cosmic strings with such values of $G \mu$ would be beyond the reach of LIGO, but could be measured by other planned and proposed detectors such as SKA, LISA, TianQin, AION-1 km, AEDGE, Einstein Telescope, and Cosmic Explorer.

DOI: 10.1103/PhysRevLett.126.041304

Introduction.-Stimulated by the direct discovery of gravitational waves (GWs) by the LIGO and Virgo Collaborations [1-8] of black holes and neutron stars at frequencies $f \gtrsim 10 \mathrm{~Hz}$, there is widespread interest in experiments exploring other parts of the GW spectrum. Foremost among these are pulsar timing array (PTA) experiments, which are sensitive to GWs with frequencies $f \lesssim 1 /$ yr. PTA experiments probe the possible existence of a stochastic GW background (SGWB), as might be generated by very different physical phenomena such as astrophysical sources of GWs, e.g., the mergers of supermassive black hole (SMBHs), or cosmological sources, e.g., cosmic strings.

Aggregating pulsar measurements for over a decade, the EPTA [9], PPTA [10], and NANOGrav [11] PTA experiments have pushed their sensitivities down to an energy density $\Omega_{\mathrm{GW}} h^{2} \lesssim 10^{-9}$ over frequencies in the range $f \in\left(2.5 \times 10^{-9}, 1.2 \times 10^{-8}\right) \mathrm{Hz}$. Until recently, there has been no indication of a positive signal above background. However, a recent NANOGrav analysis of $12.5 \mathrm{yr}$ of pulsar timing data [12] reports strong evidence for a stochastic common-spectrum process that may be interpreted as a GW signal with amplitude $A \sim \mathcal{O}\left(10^{-15}\right)$ at $f \sim 1 / \mathrm{yr}$. The NANOGrav Collaboration notes that this signal is in apparent tension with previous upper limits on the SGWB in this frequency range, but argues that this is

Published by the American Physical Society under the terms of the Creative Commons Attribution 4.0 International license. Further distribution of this work must maintain attribution to the author(s) and the published article's title, journal citation, and DOI. not real, but reflects its improved treatment of the intrinsic pulsar red noise. The NANOGrav signal does not exhibit significant monopole or dipole correlations, as might arise, e.g., from reference clock or solar-system ephemeris systematics, respectively. On the other hand, neither does the signal exhibit significant quadrupole correlations, which would have been a "smoking gun" for a GW background, and the NANOGrav Collaboration does not claim a detection of GWs.

Nevertheless, we are emboldened to explore the implications of this possible SGWB detection by NANOGrav for cosmic string models, discussing how experiments could confirm or disprove such an interpretation. Upper limits on the SGWB are often quoted assuming a spectrum described by a GW abundance proportional to $f^{2 / 3}$, as expected for SMBH mergers [13]. However, the cosmic string GW spectrum is not a simple power law, but is convex with an amplitude and a frequency-dependent slope that depend on the parameter, $G \mu$, where $G$ is the Newton constant of gravitation and $\mu$ is the string tension. Any limit (or estimate) of $G \mu$ from any specific experiment must take into account take into account the appropriate slope parameter, which is in general $\neq 2 / 3$ in the characteristic frequency measurement range. Once an allowed (interesting) value of $G \mu$ has been identified, however, the cosmic string prediction for the magnitude and spectral shape of the SGWB is then fixed as a function of frequency, and can then be compared with the sensitivities of other experiments.

In this paper we calculate the effective slope parameter for the timing-residual cross-power spectral density $\gamma$ (which translates to $\gamma=5-\beta$ for $\Omega \propto f^{\beta}$ ) for frequencies in the range $\left(2.5 \times 10^{-9}, 1.2 \times 10^{-8}\right) \mathrm{Hz}$ used in Ref. [12] 
to make a single-power fit to the NANOGrav $12.5 \mathrm{yr}$ data. The best fit to the NANOGrav data is shown as an orange dashed line in the left panel of Fig. 1 of Ref. [12], and the $68 \%$ and $95 \%$ C.L. ranges in the $(\gamma, A)$ plane are shown as orange dashed and dotted ellipses in the right panel of Fig. 1 of Ref. [12]. We find that the cosmic string model gives a better fit than does a single power law with $\gamma=13 / 3$ as suggested by models of SMBH mergers: the one-parameter cosmic string prediction crosses the $68 \%$ CL ellipse, whereas the $\gamma=13 / 3$ line passes outside it though within the $95 \%$ ellipse. The GW spectra predicted by the cosmic string model for $G \mu \in\left(2 \times 10^{-11}, 2 \times 10^{-10}\right)$, the range where it lies within the NANOGrav $12.5 \mathrm{yr}$ 95\% C.L. region in the $(\gamma, A)$ plane, are all completely compatible with the EPTA upper limit, although some tension with the PPTA results remains in the upper part of our range. The cosmic string predictions are well within the estimated reaches of the SKA [14], LISA [15,16], TianQin [17,18], AEDGE [19], AION-1 km [20], ET [21,22] and CE [23] experiments, but beyond the present and estimated future sensitivities of the LIGO [24-27] experiment.

$G W$ spectrum from cosmic strings.-Cosmic strings are one-dimensional stable objects described by their characteristic tension $\mu$. They are a common prediction of many extensions of the standard model [28,29] featuring a $U(1)$ symmetry-breaking phase transition in the early Universe [30]. They can also arise in superstring theory as cosmologically stretched fundamental strings [31,32]. We focus mostly on the former case, for which the intercommutation probability $p$ (the probability that strings reconnect in a different way after crossing) takes the value $p=1$, and comment on this choice towards the end of the following section.

We use a simple method of computation of the GW spectrum from a cosmic string network following [33,34] (for an overview, see [35]). We utilize the velocitydependent one-scale (VOS) model [36-38], assuming that the length of a loop produced by the network $\ell$ at time $t_{i}$ evolves as

$$
\ell=\alpha_{\ell} t_{i}-\Gamma G \mu\left(t-t_{i}\right),
$$

where $G \mu$ is the string tension and $\alpha_{\ell}$ the initial loop size. Following the guidance from recent numerical simulations $[39,40]$, we focus on the largest loops produced by the network, fixing $\alpha_{\ell}=0.1$, as these dominate the $\mathrm{GW}$ emission. String loops emit at normal oscillation mode frequencies, allowing us to express the frequency measured today from mode $k$ with emission time $\tilde{t}$ as

$$
f=\frac{a(\tilde{t})}{a\left(t_{0}\right)} \frac{2 k}{\alpha_{\ell} t_{i}-\Gamma G \mu\left(\tilde{t}-t_{i}\right)},
$$

where $t_{0}$ is the current time. The GW abundance can be computed as a sum over individual emission modes

$$
\Omega_{\mathrm{GW}}^{\mathrm{CS}}(f)=\sum_{k=1}^{\infty} k \Gamma^{(k)} \Omega_{\mathrm{GW}}^{(k)}(f),
$$

where the total emission rate $\Gamma$ is found in simulations to have the value $\Gamma \simeq 50$ [39-43], and we assume that this is dominated by emission from cusps with $\Gamma^{(k)}=$ $\Gamma k^{-(4 / 3)} /\left(\sum_{m=1}^{\infty} m^{-(4 / 3)}\right)$ [34]. We truncate the sum in Eq. (3) at $10^{3}$ modes, beyond which we approximate it with an integral that guarantees good accuracy also for the high-frequency part of the spectrum [44-46]. The contribution of each emission mode in Eq. (3) has the form (see Ref. [34] for details)

$$
\begin{aligned}
\Omega_{\mathrm{GW}}^{(k)}(f)= & \frac{16 \pi}{3 H_{0}^{2}} \frac{(0.1)(G \mu)^{2}}{\alpha_{\ell}\left(\alpha_{\ell}+\Gamma G \mu\right)} \frac{1}{f} \\
& \times \int_{t_{F}}^{t_{0}} d \tilde{t} \frac{C_{\mathrm{eff}}\left(t_{i}\right)}{t_{i}^{4}}\left(\frac{a(\tilde{t})}{a\left(t_{0}\right)}\right)^{5}\left(\frac{a\left(t_{i}\right)}{a(\tilde{t})}\right)^{3} \Theta\left(t_{i}-t_{F}\right) .
\end{aligned}
$$

In evaluating the scale factor $a(t)$, we use the number of degrees of freedom predicted by the standard model as given by microMEGAS [47]. The lower integration limit $t_{F}$ corresponds to the network formation time, which can be assumed to be an arbitrarily small number for our purposes, as it only controls the high frequency cutoff of the spectrum, whereas we are mostly interested in the lowfrequency peak. [48] We calculate the $C_{\text {eff }}$ factor controlling the loop number density in Eq. (4) using the velocitydependent one-scale (VOS) [36-38,51,52] model as in Refs. [33,34] which gives $C_{\text {eff }}=5.4$ and 0.39 during radiation and matter domination, respectively. These values agree quite well with the values predicted by recent numerical simulations [39,40,53-55]. Finally the additional factor 0.1 comes from the same simulations, which find that only this fraction of energy goes into large loops that produce GWs efficiently, whereas the rest goes into the kinetic energy of small loops that is then lost to redshifting.

Connection with experimental results.-The most recent experimental results from $12.5 \mathrm{yr}$ of NANOGrav data [12] are expressed in terms of a generic power-law signal with characteristic strain given by

$$
h_{c}(f)=A\left(\frac{f}{f_{\mathrm{yr}}}\right)^{\alpha},
$$

where $f_{\mathrm{yr}}=1 \mathrm{yr}^{-1}$. The abundance of gravitational waves has the standard form, which can also be recast as a power law:

$\Omega(f)=\frac{2 \pi^{2}}{3 H_{0}^{2}} f^{2} h_{c}(f)^{2}=\Omega_{\mathrm{yr}}\left(\frac{f}{f_{\mathrm{yr}}}\right)^{\beta}=\Omega_{\mathrm{yr}}\left(\frac{f}{f_{\mathrm{yr}}}\right)^{5-\gamma}$,

where 


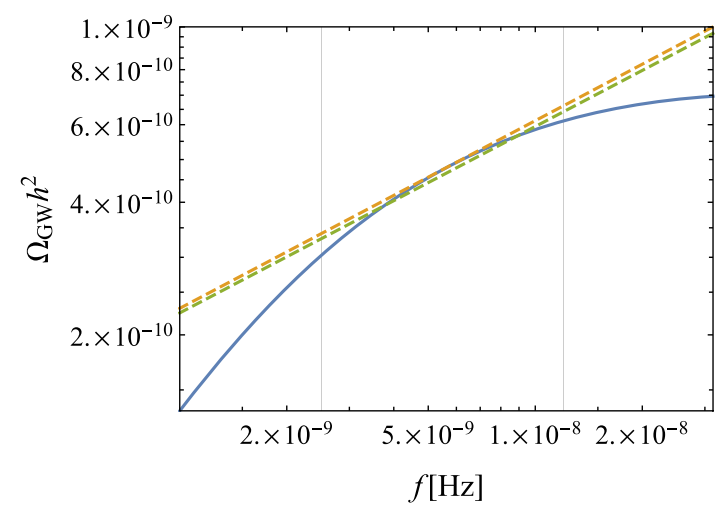

FIG. 1. Cosmic string spectra (solid blue curves) together with our fitted power laws for $G \mu=4 \times 10^{-11}$. The green-dashed lines show the results of numerically fitting the curves, while the orange lines result from the simple logarithmic derivative in Eq. (8). The thin gray lines indicate the frequency range of interest that was used in the NANOGrav linear fit.

$$
\Omega_{\mathrm{yr}}=\frac{2 \pi^{2}}{3 H_{0}^{2}} A^{2} f_{\mathrm{yr}}^{2}
$$

The experimental analysis was cast in terms of the power law found in the timing-residual cross-power spectral density $\gamma=3-2 \alpha=5-\beta$, and we adopt this notation.

In order to make connection with the experimental results, we approximate the cosmic string spectra with power laws in the range of frequencies where the possible signal was observed. The simple power-law approximation used by NANOGrav [12] was fitted to 5 bins covering roughly $f \in\left(2.5 \times 10^{-9}, 1.2 \times 10^{-8}\right) \mathrm{Hz}$, with the higherfrequency bins still seemingly dominated by noise in the data. To estimate the prospective cosmic string signal for any given value of $G \mu$, we fit numerically a power law, see Eq. (6), to the calculation of the spectrum described above in the range of interest. We show an example of this fit for $G \mu=4 \times 10^{-11}$ in Fig. 1. However, as also as we also see in the plot, we find that a very good approximation is obtained by simply taking a logarithmic derivative of our cosmic string spectrum to find the slope

$$
\begin{aligned}
& \gamma=5-\left.\frac{d \log \Omega_{\mathrm{GW}}^{\mathrm{CS}}(f)}{d \log f}\right|_{f=f_{*}}, \\
& A=\sqrt{\frac{3 H_{0}^{2}}{2 \pi^{2}} \frac{\Omega_{\mathrm{GW}}^{\mathrm{CS}}\left(f_{*}\right)\left(f_{\mathrm{yr}} / f_{*}\right)^{5-\gamma}}{f_{\mathrm{yr}}^{2}}},
\end{aligned}
$$

at the reference frequency $f_{*} \approx 5.6 \times 10^{-9} \mathrm{~Hz}$.

We show in Fig. 2 the resulting values of $\gamma$ and $A$ for a range of $G \mu$ values of interest overlaid on the NANOGrav fit to their $12.5 \mathrm{yr}$ data [12]. We find that values of the string tension $G \mu \in\left(4 \times 10^{-11}, 10^{-10}\right)$ give results within the $68 \% \mathrm{CL}$ range of the NANOgrav fit, while $G \mu \in$ $\left(2 \times 10^{-11}, 3 \times 10^{-10}\right)$ make predictions within the $95 \%$

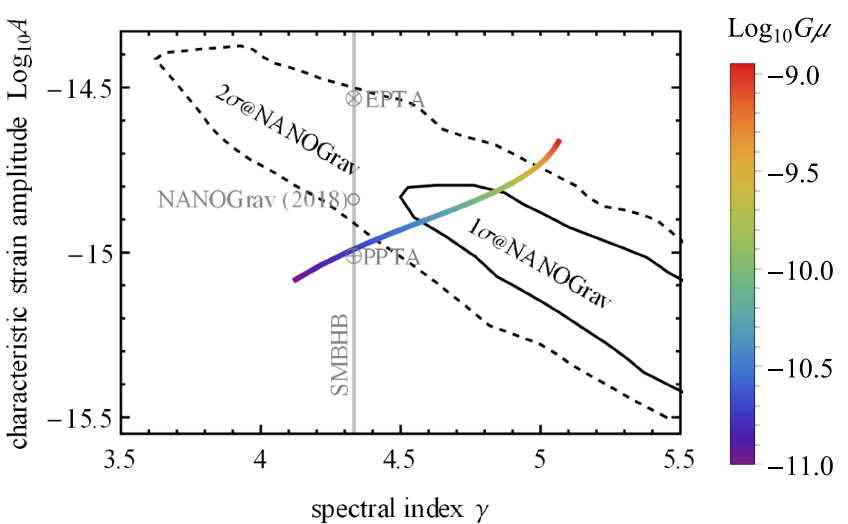

FIG. 2. The curve shows the slope $\gamma$ and amplitude $A$ of a power-law signal approximating the calculated cosmic string spectra, see Eqs. (6) and (8), with $G \mu$ values indicated by the indicated rainbow colors in the indicated frequency range. The solid and dashed black lines indicate the $68 \%$ and $95 \%$ ranges of $(\gamma, A)$ fitted to their $12.5 \mathrm{yr}$ data by the NANOGrav Collaboration [12]. The gray vertical line at $\gamma=13 / 3$ represents the slope expected for SMBH mergers [13], while the points on it mark the upper limits on the amplitude from previously reported pulsar timing data for that spectrum.

range. Interestingly, the cosmic string interpretation offers a slightly better fit than SMBH mergers, which predict $\gamma=13 / 3$ (shown as the vertical gray line in Fig. 2) yielding a fit that is at best within the $95 \%$ C.L. range but outside the $68 \%$ range.

The new NANOGrav $12.5 \mathrm{yr}$ [12] results are in some tension with previous bounds from PPTA [10] and a previous NANOGrav analysis of their $11 \mathrm{yr}$ data [11], though compatible with EPTA data [9]. Figure 3 shows a comparison of the older constraints with the cosmic string

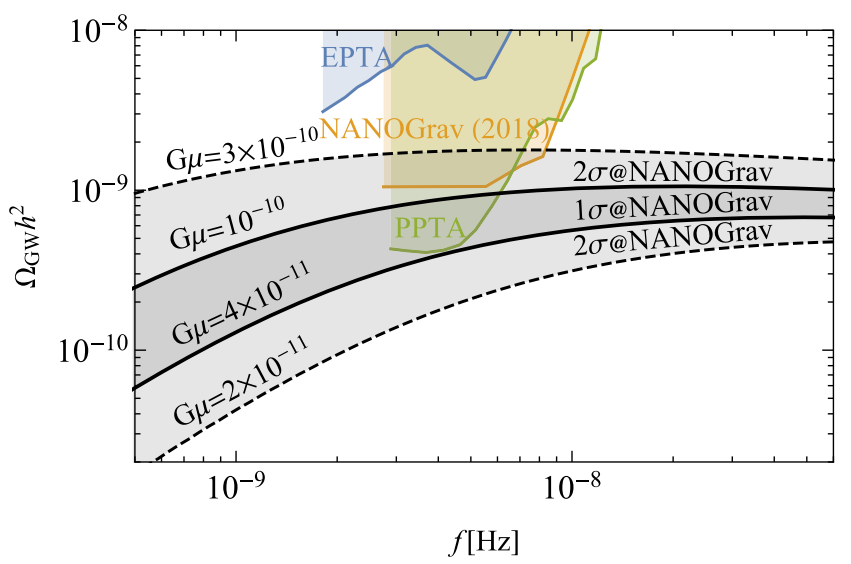

FIG. 3. Cosmic string spectra calculated for $\left.f \in 5 \times 10^{-10}, 6 \times 10^{-8}\right)$ with $G \mu \in\left(4 \times 10^{-11}, 10^{-10}\right)$ (between the solid black lines) and $G \mu \in\left(2 \times 10^{-11}, 3 \times 10^{-10}\right)$ (between the dashed black lines) that fit the NANOGrav $12.5 \mathrm{yr}$ data within the $68 \%$ and $95 \%$ confidence levels, respectively. We also show previously reported bounds from PPTA [10], EPTA [9], and NANOGrav 11 yr data [11]. 


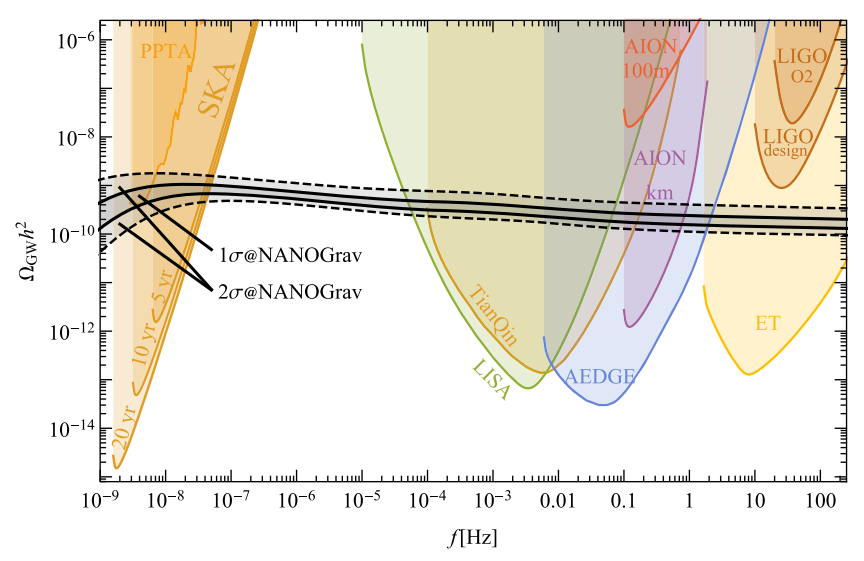

FIG. 4. Cosmic string spectra calculated for $f \in$ $\left(10^{-9}, 200\right) \mathrm{Hz}$ with $G \mu \in\left(4 \times 10^{-11}, 10^{-10}\right)$ (between the solid black lines) and $G \mu \in\left(2 \times 10^{-11}, 3 \times 10^{-10}\right)$ (between the dashed black lines) that fit the NANOGrav $12.5 \mathrm{yr}$ data at the $68 \%$ and $95 \%$ confidence levels, respectively. We also show the current sensitivity of LIGO $\mathrm{O} 2$ as well as its design sensitivity, as well as the estimated reaches of the other planned and proposed experiments SKA, LISA, TianQin, AEDGE, AION, ET, and CE.

spectra that provide $68 \%$ and $95 \%$ C.L. fits to the NANOGrav $12.5 \mathrm{yr}$ data. The apparent tension is also visible in Fig. 2, which shows previous PPTA and NANOGrav upper limits on the amplitude of a $\gamma=13 / 3$ SMBH merger spectrum (vertical gray line) from the earlier pulsar timing data releases cited above. According to the NANOGrav Collaboration [12], their new analysis uses improved priors for the intrinsic pulsar red noise (see Ref. [56] for a recent discussion). Applying these new priors to older data would ease the previous constraints and tend to reduce the tension.

Figure 4 shows the spectra that fit the new NANOGrav data at the $68 \%$ and $95 \%$ C.L.s over an extended frequency range $f \in\left(10^{-9}, 200\right) \mathrm{Hz}$. We also show the current sensitivity of LIGO O2 [27] together with its design sensitivity goal [24-26], as well as the projected sensitivities of SKA [14] and the upcoming GW experiments LISA [15,16], TianQin [17,18], AEDGE [19], AION/MAGIS $[20,57,58]$, and ET $[21,22]$. We see all the next-generation GW experiments should be able to observe cosmic string signals strong enough to fit the current NANOGrav data. However, LIGO would, unfortunately not be able to observe such a signal even after reaching its design sensitivity [59].

We have focused throughout this section on cosmic strings that always interchange partners upon crossing, so that the intercommutation probability $p=1$, though this could be reduced if the strings originated from superstring theory [31,32]. In a first approximation this just corresponds to the density of strings increasing as $p^{-1}$ for any given value of the tension, which leads to a similar increase in the amplitude of the GW signal $[64,65]$. As a result, the cosmic string curve in Fig. 2 would simply move up in amplitude as $A \propto \sqrt{\Omega} \propto \sqrt{p^{-1}}$. Since the rainbow curve passes close to the top of the NANOGrav $68 \%$ C.L. region, there is little scope for decreasing $p$ while maintaining consistency at the $68 \%$ C.L., with $\Omega_{\mathrm{GW}} h^{2}$ increasing by $<50 \%$. We note, however, that the dependence of the density on the probability can be milder than the simple $p^{-1}$ assumption [66], and that the final result is still a matter of debate [35], so that this conclusion may need to be relaxed.

Before proceeding to our conclusions, we first mention briefly other possible sources that could potentially fit the new NANOGrav data. One possibility is SMBH mergers. However, their rate is uncertain and, as already noted, a simple model led to the prediction $\gamma=13 / 3$ [13] that is apparently disfavored by the NANOGrav data, though this is sensitive to the priors used in the data analysis $[56,67]$. Another possibility is primordial inflation [15,68], which leads generically to a flat spectrum with $\gamma=5$ whose magnitude is constrained by CMB measurements [69] at $f_{\mathrm{CMB}} \approx 10^{-17} \mathrm{~Hz}$ to be orders of magnitude below the amplitude of the observed signal. The inflationary spectrum would therefore require modification if it is to fit the observed abundance at PTA frequencies. This requires $\beta \simeq 0.68$ [70], which gives a spectrum at PTA frequencies with $\gamma \simeq 4.32$, a value very close to the SMBH merger prediction [13] and again seemingly slightly disfavoured by the current data. A third possibility is a signal from a firstorder phase transition in the early Universe. However, such a signal typically peaks at a much higher frequency [71,72]. Lowering the frequency requires a transition at a lower temperature, which is possible only in a model with a hidden sector decoupled from the standard model [73], since the frequency cannot be lowered by supercooling $[74,75]$, and models coupling to the standard model with such low mass scales would already be observed. Even if a hidden sector model is capable of accommodating a very strong phase transition at a very low temperature, one expects a spectrum at PTA frequencies which has a lowfrequency slope $\beta=3$ [76] and hence $\gamma=2$, which is disfavored by the data. While some exceptions from that scaling exist, they require either an extremely strong transition [77] or modification of cosmological expansion [78], both of which would be extremely difficult to realize at low temperatures without violating other bounds.

Conclusions.-We have analysed the GW spectra produced by cosmic string networks, recasting them numerically as power laws in the frequency range $f \in$ $\left(2.5 \times 10^{-9}, 1.2 \times 10^{-8}\right) \mathrm{Hz}$ of interest to PTA experiments. This allowed us to express the resulting amplitude and slope as functions of the only free parameter in our model, which is the string tension $G \mu$. We then use these results to make contact with the recent NANOGrav $12.5 \mathrm{yr}$ [12] data release, which finds evidence of a stochastic common-spectrum process, analysed in terms of power-law modeling, that could be interpreted as a $\mathrm{GW}$ background. We find that a cosmic string tension 
$G \mu \in\left(4 \times 10^{-11}, 10^{-10}\right)$ fits the data within the $68 \%$ C.L. region around the best fit while $G \mu \in\left(2 \times 10^{-11}\right.$, $\left.3 \times 10^{-10}\right)$ is compatible with the data at the $95 \%$ C.L. Cosmic strings provide a better fit to the current data than a GW spectrum from SMBH mergers, which can fit the data at the $95 \%$ C.L. but not the $68 \%$ C.L. We also show all next-generation GW detectors including SKA, LISA, TianQin, AEDGE, AION, and ET will be able to probe the cosmic string spectra that fit the current data, whereas LIGO seems unlikely be able to probe them in the absence of additional cosmological or model features.

A key probe of any GW interpretation of the NANOGrav data would be the appearance of quadrupole correlations, which have not (yet) been detected. Beyond this, measurement of a SGWB background compatible with the shape of spectrum shown in Fig. 4 over a large range of frequencies would provide crucial confirmation of our bold GW interpretation of the NANOGrav $12.5 \mathrm{yr}$ data.

This work was supported by the UK STFC Grant No. ST/P000258/1. J. E. also acknowledges support from the Estonian Research Council Grant No. MOBTT5, and M. L. from the Polish National Science Center Grant No. 2018/31/D/ST2/02048. The project is co-financed by the Polish National Agency for Academic Exchange within Polish Returns Programme under Agreement No. PPN/ PPO/2020/1/00013/U/00001.

Note added in proof.-For completeness, we note that other models have also been proposed as possible explanations of the NANOGrav data, including Refs. [79] and [80], which also deal with cosmic strings, as well as explanations involving primordial black hole production [81], cosmological phase transitions [82], audible axions [83], inflation [84], domain walls [85], and possible violation of the null energy condition [86].

*john.ellis@cern.ch

†marek.lewicki@kcl.ac.uk

[1] B. Abbott et al. (LIGO Scientific, Virgo Collaborations), Observation of Gravitational Waves from a Binary Black Hole Merger, Phys. Rev. Lett. 116, 061102 (2016).

[2] B. P. Abbott et al. (LIGO Scientific, Virgo Collaborations), GW151226: Observation of Gravitational Waves from a 22-Solar-Mass Binary Black Hole Coalescence, Phys. Rev. Lett. 116, 241103 (2016).

[3] B. P. Abbott et al. (Virgo, LIGO Scientific Collaborations), GW170104: Observation of a 50-Solar-Mass Binary Black Hole Coalescence at Redshift 0.2, Phys. Rev. Lett. 118, 221101 (2017).

[4] B. P. Abbott et al. (LIGO Scientific, Virgo Collaborations), GW170608: Observation of a 19-solar-mass binary black hole coalescence, Astrophys. J. 851, L35 (2017).

[5] B. Abbott et al. (LIGO Scientific, Virgo Collaborations), GW170814: A Three-Detector Observation of Gravitational
Waves from a Binary Black Hole Coalescence, Phys. Rev. Lett. 119, 141101 (2017).

[6] B. Abbott et al. (Virgo, LIGO Scientific Collaborations), GW170817: Observation of Gravitational Waves from a Binary Neutron Star Inspiral, Phys. Rev. Lett. 119, 161101 (2017).

[7] R. Abbott et al. (LIGO Scientific, Virgo Collaborations), GW190814: Gravitational waves from the coalescence of a 23 solar mass black hole with a 2.6 solar mass compact object, Astrophys. J. Lett. 896, L44 (2020).

[8] R. Abbott et al. (LIGO Scientific, Virgo Collaborations), GW190521: A Binary Black Hole Merger with a Total Mass of $150 M_{\odot}$, Phys. Rev. Lett. 125, 101102 (2020).

[9] L. Lentati et al., European pulsar timing array limits on an isotropic stochastic gravitational-wave background, Mon. Not. R. Astron. Soc. 453, 2577 (2015).

[10] R. M. Shannon et al., Gravitational waves from binary supermassive black holes missing in pulsar observations, Science 349, 1522 (2015).

[11] Z. Arzoumanian et al. (NANOGrav Collaboration), The NANOGrav 11-year data set: Pulsar-timing constraints on the stochastic gravitational-wave background, Astrophys. J. 859, 47 (2018).

[12] Z. Arzoumanian et al. (NANOGrav Collaboration), The NANOGrav 12.5-year data set: Search for an isotropic stochastic gravitational-wave background, arXiv:2009.04496.

[13] E. Phinney, A Practical theorem on gravitational wave backgrounds, arXiv:astro-ph/0108028.

[14] G. Janssen et al., Gravitational wave astronomy with the SKA, Proc. Sci. AASKA14 (2015) 037 [arXiv:1501.00127].

[15] N. Bartolo et al., Science with the space-based interferometer LISA. IV: Probing inflation with gravitational waves, J. Cosmol. Astropart. Phys. 12 (2016) 026.

[16] C. Caprini, D. G. Figueroa, R. Flauger, G. Nardini, M. Peloso, M. Pieroni, A. Ricciardone, and G. Tasinato, Reconstructing the spectral shape of a stochastic gravitational wave background with LISA, J. Cosmol. Astropart. Phys. 11 (2019) 017.

[17] J. Luo et al. (TianQin Collaboration), TianQin: A spaceborne gravitational wave detector, Classical Quantum Gravity 33, 035010 (2016).

[18] J. Mei et al., The TianQin project: Current progress on science and technology, Prog. Theor. Exp. Phys. (2020).

[19] Y. A. El-Neaj et al. (AEDGE Collaboration), AEDGE: Atomic experiment for dark matter and gravity exploration in space, Eur. Phys. J. Quantum Technol. 7, 6 (2020).

[20] L. Badurina et al., AION: An atom interferometer observatory and network, J. Cosmol. Astropart. Phys. 05 (2020) 011.

[21] M. Punturo et al., The Einstein telescope: A third-generation gravitational wave observatory, Classical Quantum Gravity 27, 194002 (2010).

[22] S. Hild et al., Sensitivity studies for third-generation gravitational wave observatories, Classical Quantum Gravity 28, 094013 (2011).

[23] B. P. Abbott et al. (LIGO Scientific Collaboration), Exploring the sensitivity of next generation gravitational wave detectors, Classical Quantum Gravity 34, 044001 (2017).

[24] J. Aasi et al. (LIGO Scientific Collaboration), Advanced LIGO, Classical Quantum Gravity 32, 115012 (2015). 
[25] E. Thrane and J. D. Romano, Sensitivity curves for searches for gravitational-wave backgrounds, Phys. Rev. D 88, 124032 (2013).

[26] B. P. Abbott et al. (Virgo, LIGO Scientific Collaborations), GW150914: Implications for the Stochastic Gravitational Wave Background from Binary Black Holes, Phys. Rev. Lett. 116, 131102 (2016).

[27] B. Abbott et al. (LIGO Scientific, Virgo Collaborations), Search for the isotropic stochastic background using data from Advanced LIGO's second observing run, Phys. Rev. D 100, 061101 (2019).

[28] R. Jeannerot, J. Rocher, and M. Sakellariadou, How generic is cosmic string formation in SUSY GUTs, Phys. Rev. D 68, 103514 (2003).

[29] S. F. King, S. Pascoli, J. Turner, and Y.-L. Zhou, Gravitational Waves and Proton Decay: Complementary Windows into Grand Unified Theories, Phys. Rev. Lett. 126, 021802 (2021).

[30] H. B. Nielsen and P. Olesen, Vortex line models for dual strings, Nucl. Phys. B61, 45 (1973).

[31] G. Dvali and A. Vilenkin, Formation and evolution of cosmic D strings, J. Cosmol. Astropart. Phys. 03 (2004) 010.

[32] E. J. Copeland, R. C. Myers, and J. Polchinski, Cosmic F and $D$ strings, J. High Energy Phys. 06 (2004) 013.

[33] Y. Cui, M. Lewicki, D. E. Morrissey, and J. D. Wells, Cosmic archaeology with gravitational waves from cosmic strings, Phys. Rev. D 97, 123505 (2018).

[34] Y. Cui, M. Lewicki, D. E. Morrissey, and J. D. Wells, Probing the pre-BBN universe with gravitational waves from cosmic strings, J. High Energy Phys. 01 (2019) 081.

[35] P. Auclair et al., Probing the gravitational wave background from cosmic strings with LISA, J. Cosmol. Astropart. Phys. 04 (2020) 034.

[36] C. J. A. P. Martins and E. P. S. Shellard, String evolution with friction, Phys. Rev. D 53, R575 (1996).

[37] C. J. A. P. Martins and E. P. S. Shellard, Quantitative string evolution, Phys. Rev. D 54, 2535 (1996).

[38] C. J. A. P. Martins and E. P. S. Shellard, Extending the velocity dependent one scale string evolution model, Phys. Rev. D 65, 043514 (2002).

[39] J. J. Blanco-Pillado, K. D. Olum, and B. Shlaer, The number of cosmic string loops, Phys. Rev. D 89, 023512 (2014).

[40] J. J. Blanco-Pillado and K. D. Olum, Stochastic gravitational wave background from smoothed cosmic string loops, Phys. Rev. D 96, 104046 (2017).

[41] A. Vilenkin, Gravitational radiation from cosmic strings, Phys. Lett. 107B, 47 (1981).

[42] N. Turok, Grand unified strings and galaxy formation, Nucl. Phys. B242, 520 (1984).

[43] J. M. Quashnock and D. N. Spergel, Gravitational selfinteractions of cosmic strings, Phys. Rev. D 42, 2505 (1990).

[44] Y. Cui, M. Lewicki, and D. E. Morrissey, Gravitational Wave Bursts as Harbingers of Cosmic Strings Diluted by Inflation, Phys. Rev. Lett. 125, 211302 (2020).

[45] S. Blasi, V. Brdar, and K. Schmitz, Fingerprint of low-scale leptogenesis in the primordial gravitational-wave spectrum, Phys. Rev. Research 2, 043321 (2020).

[46] Y. Gouttenoire, G. Servant, and P. Simakachorn, Beyond the standard models with cosmic strings, J. Cosmol. Astropart. Phys. 07 (2020) 032.
[47] G. Bélanger, F. Boudjema, A. Goudelis, A. Pukhov, and B. Zaldivar, micrOMEGAs5.0: Freeze-in, Comput. Phys. Commun. 231, 173 (2018).

[48] In fact, in generic cases a much more important cut-off on the high-frequency end of the spectrum appears where particle emission becomes more important than GW emission $[49,50]$.

[49] P. Auclair, D. A. Steer, and T. Vachaspati, Particle emission and gravitational radiation from cosmic strings: Observational constraints, Phys. Rev. D 101, 083511 (2020).

[50] D. Matsunami, L. Pogosian, A. Saurabh, and T. Vachaspati, Decay of Cosmic String Loops Due to Particle Radiation, Phys. Rev. Lett. 122, 201301 (2019).

[51] P. P. Avelino and L. Sousa, Scaling laws for weakly interacting cosmic (super)string and p-brane networks, Phys. Rev. D 85, 083525 (2012).

[52] L. Sousa and P. P. Avelino, Stochastic gravitational wave background generated by cosmic string networks: Velocitydependent one-scale model versus scale-invariant evolution, Phys. Rev. D 88, 023516 (2013).

[53] J. J. Blanco-Pillado, K. D. Olum, and B. Shlaer, Large parallel cosmic string simulations: New results on loop production, Phys. Rev. D 83, 083514 (2011).

[54] J. J. Blanco-Pillado, K. D. Olum, and J. M. Wachter, Energy-conservation constraints on cosmic string loop production and distribution functions, Phys. Rev. D 100, 123526 (2019).

[55] J. J. Blanco-Pillado and K. D. Olum, Direct determination of cosmic string loop density from simulations, Phys. Rev. D 101, 103018 (2020).

[56] J. S. Hazboun, J. Simon, X. Siemens, and J. D. Romano, Model dependence of bayesian gravitational-wave background statistics for pulsar timing arrays, arXiv:2009.05143.

[57] P. W. Graham, J. M. Hogan, M. A. Kasevich, and S. Rajendran, Resonant mode for gravitational wave detectors based on atom interferometry, Phys. Rev. D 94, 104022 (2016).

[58] P. W. Graham, J. M. Hogan, M. A. Kasevich, S. Rajendran, and R. W. Romani (MAGIS Collaboration), Mid-band gravitational wave detection with precision atomic sensors, arXiv: 1711.02225 .

[59] However, LIGO could potentially probe spectra fitting the data in alternative models with additional features due, e.g., to modification of the spectrum by non-standard cosmological expansion $[33,34,46]$, or cosmic string models featuring large production of very small scale loops [60-63].

[60] L. Lorenz, C. Ringeval, and M. Sakellariadou, Cosmic string loop distribution on all length scales and at any redshift, J. Cosmol. Astropart. Phys. 10 (2010) 003.

[61] C. Ringeval and T. Suyama, Stochastic gravitational waves from cosmic string loops in scaling, J. Cosmol. Astropart. Phys. 12 (2017) 027.

[62] P. Auclair, C. Ringeval, M. Sakellariadou, and D. Steer, Cosmic string loop production functions, J. Cosmol. Astropart. Phys. 06 (2019) 015.

[63] P. G. Auclair, Impact of the small-scale structure on the stochastic background of gravitational waves from cosmic strings, J. Cosmol. Astropart. Phys. 11 (2020) 050.

[64] M. Sakellariadou, A Note on the evolution of cosmic string/ superstring networks, J. Cosmol. Astropart. Phys. 04 (2005) 003 . 
[65] J. J. Blanco-Pillado, K. D. Olum, and X. Siemens, New limits on cosmic strings from gravitational wave observation, Phys. Lett. B 778, 392 (2018).

[66] A. Avgoustidis and E. Shellard, Effect of reconnection probability on cosmic (super)string network density, Phys. Rev. D 73, 041301(R) (2006).

[67] R. van Haasteren, Y. Levin, P. McDonald, and T. Lu, On measuring the gravitational-wave background using pulsar timing arrays, Mon. Not. R. Astron. Soc. 395, 1005 (2009).

[68] C. Caprini and D. G. Figueroa, Cosmological backgrounds of gravitational waves, Classical Quantum Gravity 35, 163001 (2018).

[69] N. Aghanim et al. (Planck Collaboration), Planck 2015 results. XI. CMB power spectra, likelihoods, and robustness of parameters, Astron. Astrophys. 594, A11 (2016).

[70] P. D. Lasky et al., Gravitational-Wave Cosmology Across 29 Decades in Frequency, Phys. Rev. X 6, 011035 (2016).

[71] C. Caprini et al., Science with the space-based interferometer eLISA. II: Gravitational waves from cosmological phase transitions, J. Cosmol. Astropart. Phys. 04 (2016) 001.

[72] C. Caprini et al., Detecting gravitational waves from cosmological phase transitions with LISA: An update, J. Cosmol. Astropart. Phys. 03 (2020) 024.

[73] M. Breitbach, J. Kopp, E. Madge, T. Opferkuch, and P. Schwaller, Dark, cold, and noisy: Constraining secluded hidden sectors with gravitational waves, J. Cosmol. Astropart. Phys. 07 (2019) 007.

[74] J. Ellis, M. Lewicki, and J. M. No, On the maximal strength of a first-order electroweak phase transition and its gravitational wave signal, J. Cosmol. Astropart. Phys. 04 (2019) 003.

[75] J. Ellis, M. Lewicki, J. M. No, and V. Vaskonen, Gravitational wave energy budget in strongly supercooled phase transitions, J. Cosmol. Astropart. Phys. 06 (2019) 024.

[76] C. Caprini, R. Durrer, T. Konstandin, and G. Servant, General properties of the gravitational wave spectrum from phase transitions, Phys. Rev. D 79, 083519 (2009).

[77] M. Lewicki and V. Vaskonen, Gravitational wave spectra from strongly supercooled phase transitions, Eur. Phys. J. C 80, 1003 (2020).
[78] J. Ellis, M. Lewicki, and V. Vaskonen, Updated predictions for gravitational waves produced in a strongly supercooled phase transition, J. Cosmol. Astropart. Phys. 11 (2020) 020.

[79] S. Blasi, V. Brdar, and K. Schmitz, following Letter, Has NANOGrav Found First Evidence for Cosmic Strings? Phys. Rev. Lett. 126, 041305 (2021).

[80] W. Buchmuller, V. Domcke, and K. Schmitz, arXiv: 2009.10649; R. Samanta and S. Datta, arXiv:2009.13452; S. Chigusa, Y. Nakai, and J. Zheng, arXiv:2011.04090; N. Ramberg and L. Visinelli, arXiv:2012.06882.

[81] V. Vaskonen and H. Veermäe, arXiv:2009.07832; V. De Luca, G. Franciolini, and A. Riotto, arXiv:2009.08268; K. Kohri and T. Terada, arXiv:2009.11853; S. Sugiyama, V. Takhistov, E. Vitagliano, A. Kusenko, M. Sasaki, and M. Takada, arXiv:2010.02189; G. Domènech and S. Pi, arXiv:2010.03976; S. Bhattacharya, S. Mohanty, and P. Parashari, arXiv:2010.05071.

[82] Y. Nakai, M. Suzuki, F. Takahashi, and M. Yamada, arXiv:2009.09754; A. Neronov, A. Roper Pol, C. Caprini, and D. Semikoz, arXiv:2009.14174.

[83] W. Ratzinger and P. Schwaller, arXiv:2009.11875; R. Namba and M. Suzuki, arXiv:2009.13909.

[84] S. Vagnozzi, arXiv:2009.13432; H.-H. Li, G. Ye, and Y.-S. Piao, arXiv:2009.14663; S. Kuroyanagi, T. Takahashi, and S. Yokoyama, arXiv:2011.03323.

[85] L. Bian, R.-G. Cai, J. Liu, X.-Y. Yang, and R. Zhou, arXiv:2009.13893; J. Liu, R.-G. Cai, and Z.-K. Guo, arXiv:2010.03225; C.-W. Chiang and B.-Q. Lu, arXiv: 2012.14071.

[86] H. W. H. Tahara and T. Kobayashi, arXiv:2011.01605; Y. Cai and Y.-S. Piao, arXiv:2012.11304.

Correction: The insertion of a Note added in proof was not processed properly during production, resulting in the works referred to therein being omitted from the reference section. All sources now appear in the reference section with proper citations in the Note added in proof. 